\title{
The simulated operating theatre: comprehensive training for surgical teams
}

\author{
R Aggarwal, S Undre, K Moorthy, C Vincent, A Darzi
}

Qual Saf Health Care 2004;13(Suppl 1):i27-i32. doi: 10.1136/qshc.2004.010009

Surgical excellence is traditionally defined in terms of technical performance, with little regard for the importance of interpersonal communication and leadership skills. Studies in the aviation industry have stressed the role of human factors in causing error and, in an attempt to reduce the occurrence of adverse events, led to the organisation of simulation based training scenarios. Similar strategies have recently been employed for the surgical team with the development of a simulated operating theatre project. This enables technical and nontechnical performance of the surgeon and circulating staff to be assessed by experts situated in an adjacent control room, and provides an opportunity for constructive feedback. The scenarios have good face validity and junior surgeons can benefit from the process of learning new technical skills in a realistic environment. The effect of external influences such as distractions, new technology, or a crisis scenario can also be defined, with the ultimate aim of reducing the number of adverse events arising in the real operating room.

See end of article for authors' affiliations

Correspondence to: Mr R Aggarwal, Clinical Research Fellow, Department of Surgical Oncology \& Technology, Imperial College London, 10th Floor, QEQM Building, St Mary's Hospital, Praed Street, London, W2 INY UK; rajesh.aggarwal@ imperial.ac.uk
$\mathrm{T}$ raditionally, measures of performance in the operating theatre have concentrated on assessing the skill of the surgeon alone, and more specifically technical proficiency. This has been done by assessment of time taken to complete the operation, and more recently with the use of rating scales and motion analysis systems. ${ }^{1-4}$ However, technical ability is only one of the skills required to perform a successful operation, ${ }^{5}$ with teamwork, communication, judgement, and leadership underpinning the development of surgical competence. It is then surprising that very little attention is paid to the teaching of these skills in the medical curriculum.

A similar situation existed in the aviation industry over 20 years ago, until a finding by the National Aeronautics and Space Administration (NASA) revealed that $70 \%$ of errors were due to human causes such as failed interpersonal communication, decision making and leadership. ${ }^{6}$ This led to the development of crew resource management (CRM) training which consisted of seminars, lectures, and simulator based training to enable participants to understand the limitations of human performance and develop a culture of safety. ${ }^{78}$ The success of this programme has been evident by the adoption of
CRM training in other high reliability organisations such as the military, manufacturing, and nuclear industries. ${ }^{9}$

The application of a human factors approach to errors in medicine has been driven by the anaesthetic community, with the development of anaesthesia crisis resource management (ACRM) centres across the globe. ${ }^{10}$ Trainees are provided with an opportunity to practice their skills in an artificial environment, enabling the integration of technical and team training skills. Participants also receive feedback on their performance, many of them commenting that the experience has benefited their daily practice. ${ }^{10}$ However, surgical trainees rarely receive feedback on their non-technical skills such as teamwork, judgement, and leadership until something goes wrong. Furthermore, communication skills teaching concentrates upon the doctor-patient relationship, with little regard for the skills required to develop and maintain a good relationship with fellow team members.

Adopting strategies from past developments in industry and anaesthetic simulation, the introduction of simulation based training in surgery has the potential to improve technical and nontechnical skills performance of the surgeon in the operating theatre. Similar methods can then be employed to train and assess other members of the surgical team. It is this aspect that the article shall concentrate upon, rather than a discussion of simulation strategies in other branches of medicine such as anaesthetics and intensive care. However, the overall aim of enhanced team performance and a concomitant reduction in preventable adverse events is the same.

\section{THE IMPORTANCE OF TEAMWORK IN SURGERY}

Surgical outcome has generally been determined by the patient's condition, and the technical skills of the operating surgeon. A patient's fitness for the procedure is assessed by the anaesthetic team, and can be stratified using tools such as the POSSUM (physiological and operative severity score for the enumeration of mortality and morbidity) scoring system. ${ }^{11}$ The operative skills of a surgeon are rarely assessed during real surgical procedures, even though their importance in the determination of patient outcome is obvious. Any adverse outcomes such as infection,

Abbreviations: CDR, clinical data recording; CRM, crew resource management; $\mathrm{Gl}$, gastrointestinal; NASA, National Aeronautics and Space Administration; VR, virtual reality 


\section{Key messages}

- Training in surgery currently concentrates upon acquisition of technical skills.

- There is a culture of avoidance and denial of adverse events in surgery, with disregard of the importance of non-technical skills such as interpersonal communication, judgement, leadership, and teamwork.

- The majority of errors in high reliability organisations such as aviation are due to non-technical errors.

- Simulation based training has the potential to reduce the occurrence of adverse events.

- A simulated operating theatre project enables training and assessment of technical and non-technical skills of the entire surgical team.

- Training in a simulated environment can enable the surgical team to function in a safer and more efficient manner when crises occur in real life.

bleeding, a failed procedure, or death are seen as rare, and considered an accepted risk of the procedure.

However, the technical skills of a surgeon are only one of the factors that determine surgical outcomes. ${ }^{12}$ High reliability organisations such as aviation, the military, and nuclear industries have noted the importance of a wide variety of factors in the development of a favourable outcome. These include ergonomic factors, such as the quality of interface design, team coordination and leadership, organisational culture, and quality of decision making. In a surgical context, the application of a systems approach can lead to the identification of possible sources of error which are not immediately apparent. ${ }^{12}$ These may include the use of inappropriately designed instruments, an untrained team member, repeated interruptions by ward staff, or a tired surgeon. The development of a human factors approach has led to safer performance in industry, and it is important to address these issues in the operating theatre.

In medicine, a study of anaesthetic related errors considered $80 \%$ of the occurrences to be preventable, with human error accounting for $75 \%$ of them. ${ }^{13}$ Importantly, lack of vigilance and failure to check were the most frequently reported factors associated with human error. Similar studies have been published concerning errors in drug prescribing ${ }^{14}$ and cardiac surgery, ${ }^{15}$ and the fact that many of these errors may have been preventable if suitable systems were in place to recognise them.

\section{A systems approach to error reduction}

The systems approach to understanding the surgical process and outcomes has important implications for error reduction. ${ }^{16}$ This approach accepts that humans are fallible and errors are to be expected, even in the best organisations. Countermeasures are based upon the building of defences to trap errors, and mitigation of their effects should one occur. This consists of altering the attitudes between different individuals and modifying the behavioural norms that have been established in these work settings. An example of this is the specification of theatre lists for training junior surgeons, ensuring that fewer cases are booked and thus reducing the pressure on both the surgeon and the rest of the team to complete all procedures in the allocated time.

The success of CRM training and a systems approach to error reduction in the airline industry have led to growing interest in a similar application for the surgical team. ${ }^{17}$ The operating theatre is a highly complex working environment, with different groups of individuals involved in a coordinated effort to perform highly skilled manoeuvres. This is analogous to the situation in the aeroplane and can be used as a model for the development of simulation based surgical training.

\section{Cultures and attitudes of surgical staff}

Helmreich and Davies surveyed human factors in the operating theatre and confirmed that pilots and doctors have common interpersonal problems and similarities in professional culture. ${ }^{18}$ Sexton et al quantified this by comparing responses of pilots and consultant staff to these issues. ${ }^{19}$ They collated responses from over 7000 pilots to the cockpit management attitudes questionnaire (CMAQ), and compared this with 271 consultant anaesthetists and surgeons responding to the operating room management attitudes questionnaire (ORMAQ). Eighty two per cent of consultant surgeons denied the effects of personal stress on their performance, compared to $53 \%$ of consultant anaesthetists or pilots. Perhaps more striking was the denial of fatigue on critical aspects of care by $70 \%$ of consultant surgeons, $47 \%$ of consultant anaesthetists, and only $26 \%$ of pilots. This denial of the effects of stress and fatigue may be due to a culture of working under substantial pressures and covering up mistakes. However, this is deleterious and many industrial tragedies have been linked with failure to perform appropriate actions under stress. ${ }^{20}$

Individual attitudes to teamwork varied considerably, with $97 \%$ of pilots and $84 \%$ of consultant anaesthetists advocating flat hierarchies whereby decisions are made by the team rather than an individual, compared to only 55\% of consultant surgeons. Assessment of the quality of teamwork with consultant surgeons was rated as high by $73 \%$ of surgical trainees, $28 \%$ of surgical nurses, and only $10 \%$ of anaesthetic residents. At a specialty level, 62\% of surgical staff rated teamwork with anaesthesia staff highly, whereas only $41 \%$ of anaesthesia staff rated teamwork with surgical staff highly. This reveals a relationship between perceptions of teamwork and status within the team, and can lead to differences of opinion and the emergence of unnoticed and serious errors.

This data is similar to that gathered by NASA prior to the introduction of CRM training for airline crews. The shift toward an open and non-punitive environment that accommodated questioning and recognised human limitations was a gradual but steady progression, and has led to an improvement in the attitudes and performance of cockpit crews. ${ }^{7}$

Medical personnel do seem to understand the importance of a communicative and open environment, over $80 \%$ of them reporting that pre and post operative discussions are an important part of safety and teamwork. ${ }^{21}$ However, one quarter of the group questioned are not encouraged to report their safety concerns, and only one out of three respondents felt that errors are handled appropriately at their hospital. When asked what their top recommendations were to improve patient safety, the overwhelming response was better communication.

These studies support the fact that medicine would benefit from the development of a systems approach to decrease the number of adverse events. Even if the individual is aware of a problem, there is a culture of avoidance and denial, with little regard for precaution against the fallibility of human performance.

\section{THE SIMULATED OPERATING THEATRE}

At our centre, we have developed a simulated operating theatre to pilot CRM training for surgical specialists (figure 1). This consists of a replicated operating theatre environment and an adjacent control room, separated by one way viewing 
glass. In the operating theatre is a standard operating table, diathermy and suction machines, trolleys containing suture equipment and surgical instruments, and operating room lights. A moderate fidelity anaesthetic simulator (SimMan, Laerdl, UK) consists of a mannequin which lies on the operating table and is controlled by a desktop computer in the control room. This enables the creation of a number of scenarios such as laryngospasm, hypoxia, and cardiac arrhythmias. A further trolley is available, containing standard anaesthetic equipment, tubes, and drugs.

The complete surgical team is present, consisting of an anaesthetist, anaesthetic nurse, primary surgeon, surgeon's assistant, scrub nurse, and circulating nurse. Interactions between these individuals are recorded using four ceiling mounted cameras and unobtrusively placed microphones. The multiple streams of audio and video data, together with the trace on the anaesthetic monitor, are fed into a clinical data recording (CDR) device (figure 2). This enables those present in the control room to view the data in real time, and for recordings to be made for debriefing sessions.

THE ROLE OF SIMULATION IN SURGICAL TRAINING Simulation has been defined as a device or exercise that enables the participant to reproduce or represent, under test conditions, phenomena that are likely to occur in actual performance. ${ }^{22}$ This enables repeated practice of a standardised task, and can also be used as an assessment of competence. Current surgical training programmes limit the use of simulation to technical skills training, but it is possible to extend this to training and assessment of non-technical skills during both routine and crisis scenarios. This can lead to the integration of training in technical and non-technical skills, leading to a stepwise framework for the development of surgical competence.

\section{Technical skills in surgery}

Traditionally, training in surgery has concentrated upon anatomical knowledge and the technical skills required to perform an operation. This takes the role of the apprenticeship model with a master craftsman teaching skills to a student. The apprentice practices on real cases and gradually learns to perform the procedure without supervision. Though an effective and time tested model, any mistakes that do occur are at the patient's expense. This was particularly evident with the introduction of a new surgical procedure, such as laparoscopic cholecystectomy. ${ }^{23}$

The introduction of synthetic models for surgical training attempted to ensure that the junior surgeon was armed with basic surgical skills prior to entering the operating theatre. ${ }^{24}$ Using these models, it was not only possible to make mistakes without the fear of complication, but also to repeatedly perform the same task until competence had been achieved. This was the birth of objective assessment of technical skills in surgery, and has been further enhanced with the use of surgical virtual reality systems to provide real time feedback about skill based errors. ${ }^{25}$ Incorporation of these devices in the simulated operating theatre can lead to assessment of technical skills in a realistic working environment.

\section{Non-technical skills in surgery}

However, as Spencer points out a skilfully performed operation is $75 \%$ decision making and only $25 \%$ dexterity. Decision making and other non-technical skills are not formally taught in the surgical curriculum but are rather acquired over time. In an analogous manner, it should be possible to use the simulated operating theatre environment to train and assess performance of surgical trainees at skills such as team interaction and communication. This situation will also allow surgeons to benefit from feedback, by understanding the nature and effect of their mistakes, and learn from them.

In a preliminary study, 25 surgeons of varying grades completed part of a standard varicose vein operation on a synthetic model (Limbs \& Things, Bristol, UK) which was placed over the right groin of the anaesthetic simulator. ${ }^{26}$ The complete surgical team was present, the mannequin draped as for a real procedure, and standard surgical instruments available to the operating surgeon. Video based, blinded assessment of technical skills discriminated between surgeons according to experience, though their team skills measured by two expert observers on a global rating scale

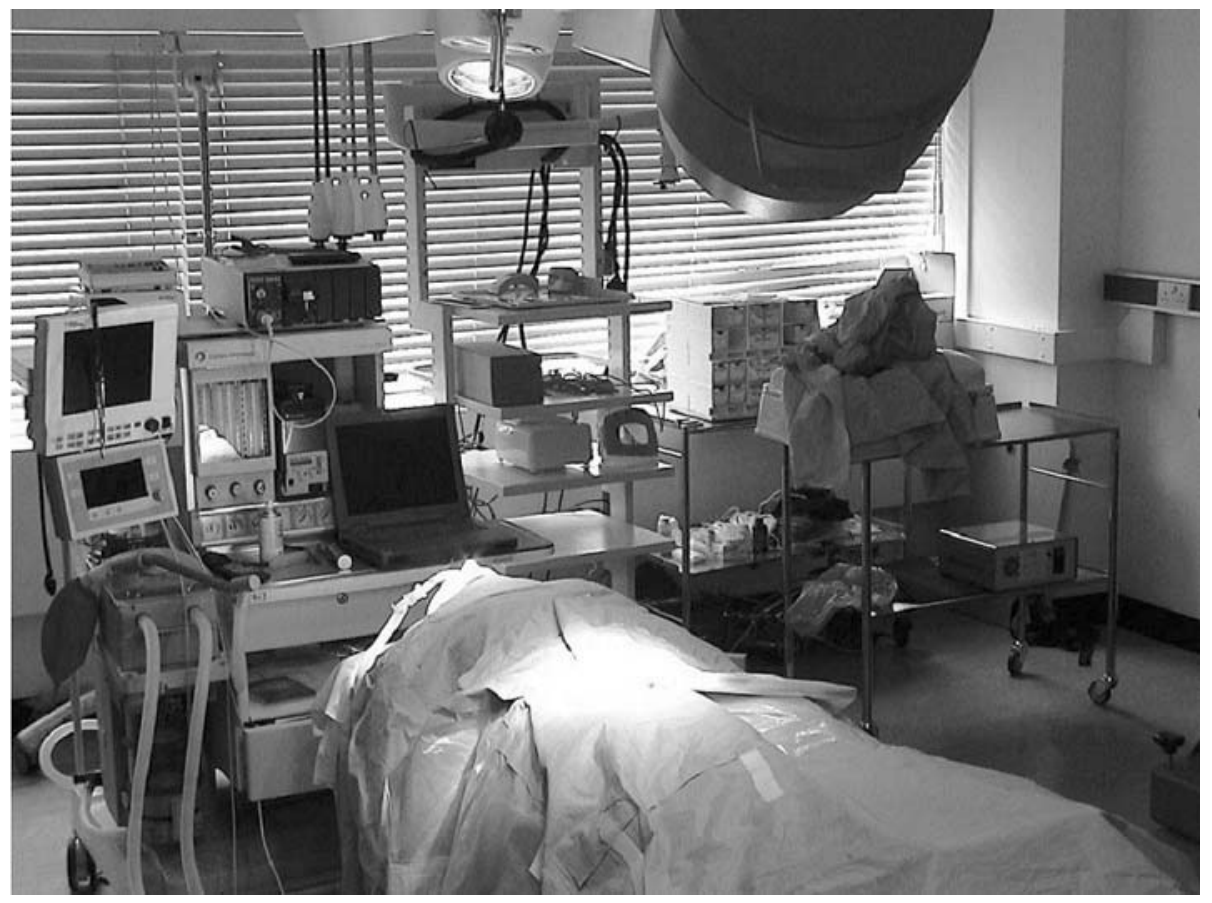

Figure 1 The simulated operating theatre. 


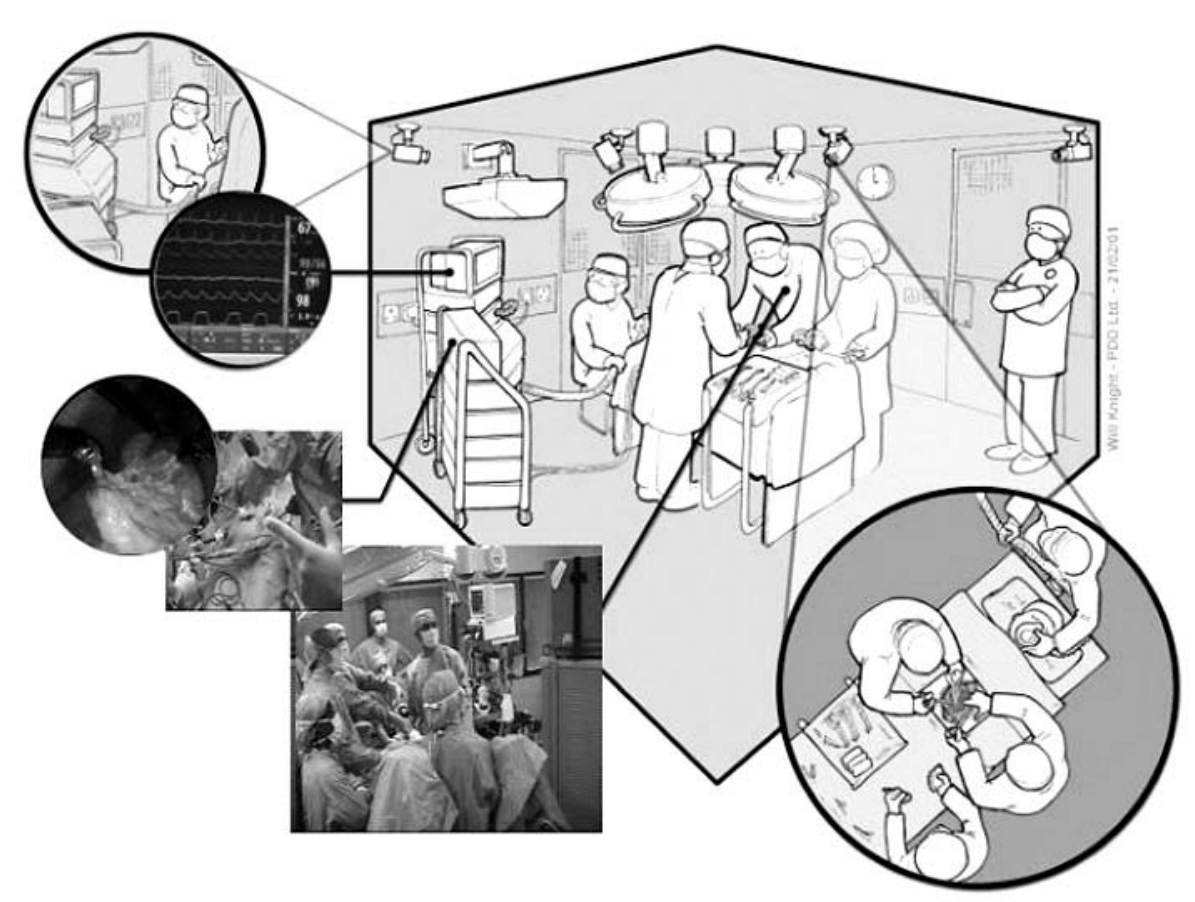

Figure 2 The clinical data recorder.

failed to show any similar differences. Many subjects did not achieve competency levels for preprocedure preparation $(90 \%)$, vigilance $(56 \%)$, team interaction $(27 \%)$, and communication $(24 \%)$. Furthermore, only two trainees positioned the patient preoperatively, and none waited for a swab/ instrument check prior to closure. Feedback responses from the participants were good, with $90 \%$ of them agreeing that the simulation was a realistic representation of an operating theatre, and $88 \%$ advocating this as a good environment for training in team skills.

\section{Assessment during crisis scenarios}

However, the greatest benefit of CRM training in aviation and anaesthetics has been for training during crisis scenarios. The varicose vein procedure was subsequently modified to include a bleeding scenario-a $5 \mathrm{~mm}$ incision was made in the "femoral vein" of the model and connected to a tube which was in turn connected to a drip bag containing simulated blood. This was controlled with a three way tap.

A further group of 10 junior and 10 senior surgical trainees were recruited to the study. ${ }^{27}$ The simulation was run as before except that, at a standardised point, the tap was opened. The trainee's technical ability to control the bleeding together with their team skills were assessed in a blinded manner by three surgeons and one human factors expert. Once again, seniors scored higher than juniors for technical skills, though there were no differences in human factors skills such as time taken to inform the team of the crisis. A majority of the participants found the model, simulated operating theatre and bleeding scenario to be realistic, with over $80 \%$ of them considering the crisis to be suitable for assessment and training of both technical and team skills. ${ }^{28}$

These studies have demonstrated the face validity of a novel crisis simulation in the simulated operating theatre, and describe how they can be used to assess the technical and non-technical performance of surgical trainees. Recent work has also introduced the notion of a ceiling effect in technical skills performance, with there being little difference between the performance of senior trainees and consultants on bench top models. ${ }^{29}$ This may be due to the limited sensitivity of the tools used to assess technical skill, or that most senior trainees have acquired the necessary technical skills required to operate competently, and that progression to expert performance is then dependant upon non-technical skills such as decision making, knowledge, and judgement.

\section{A STEPWISE APPROACH TO SURGICAL TRAINING}

While the potential of the simulated operating theatre becomes clear, it is necessary to delineate its proper place within the surgical curriculum. Haluck has suggested a stepwise framework to the mastering of skills required in surgical practice ${ }^{30}$ with many units having shown that technical skills can be learnt in a laboratory environment through the use of simulated or virtual reality (VR) models. ${ }^{31-}$ ${ }^{33}$ Surgeons are then expected to transfer their skills directly to the stressful and hostile environment of an operating theatre, and operate on a real patient. Training of their nontechnical skills is expected to occur "on the job", even though the operating theatre does not allow time for practice or reflection. Furthermore, any feedback provided to the trainee by their senior is largely subjective.

In order to address this issue, surgeons could learn their technical skills in a controlled laboratory environment, and progress to practicing them in a stressful, though safe and monitored scenario. A clear example of this is when surgical practitioners are required to perform procedures on live patients, for example during minor surgery or endoscopy. This can be a stressful experience for the trainee, as it is important not only to perform the procedure competently but also to talk to the patients, ensuring that they remain calm and comfortable. In the simulated operating theatre, it is possible to integrate technical skills training with quasiclinical scenarios using simulated patients.

This has been piloted during a two day lower gastrointestinal (GI) endoscopy course for nurse practitioners, using a computerised VR simulator (Immersion Medical, USA) ${ }^{34}$ On day one, they underwent practical training on the VR simulator in a skills laboratory, and communication skills training in the classroom. On day two, participants performed a lower GI endoscopy on a simulated patient. 
This involved an actor who is trained to play the role of the patient, lying on an examination couch, and the VR simulator placed as close to patient's buttocks as possible. Drapes covered up any joins, and a small earphone led from the simulator to the patient, instructing the patient when to voice their discomfort. This set up has been called the "virtual endoscopy suite", though is essentially the same as the simulated operating theatre.

Performance data from the VR simulator suggested an improvement in the technical skills of all participants. Concerning non-technical skills training, there were high levels of anxiety during the scenario session, though all nurses appeared to perform the procedure without conveying their anxiety to the simulated patient. They were all fully engaged in the course, and found the learning experience to have a powerful and positive impact. One of the nurses commented that the course "was in a different league from other learning experiences".

It is clear from this work that the development of surgical competence entails the acquisition of both technical and nontechnical skills. A structured training programme can begin with the acquisition of technical skills in the laboratory, using VR simulators and synthetic materials. With the development of technical competence, performance can be assessed in a simulated surgical environment and includes both technical and non-technical factors. The trainee is then allowed to progress to operating during crisis scenarios, enabling fine tuning of their skills prior to performing real procedures.

\section{CONCLUSIONS}

Competence in general surgery has generally concentrated upon the acquisition and mastery of technical skills, with little regard for the importance of skills such as team training, communication, and decision making. However, current attitudes of members of the operating team, the lack of a systems approach and the occurrence of adverse events have highlighted the need for the development of team orientated training.

The simulated operating theatre has been shown to be a realistic representation of the real operating theatre, with participants feeling immersed in the crisis scenarios. The CDR enables constructive feedback of performance in both technical and non-technical skills, and the nurse endoscopy course has underlined the presence of a powerful affective component to learning. ${ }^{35}$ The scenarios have also confirmed that the expert status of senior surgeons does not equate to better team skills when compared to junior surgeons, confirming the need to train all grades of surgeons using this approach.

Current work has concentrated upon assessing the role of the primary surgeon, though "the ultimate aim of operating room simulations should be to involve all teams to enhance overall team performance". ${ }^{36}$ Further work is planned to assess the interactions between different groups within the operating theatre, and also to assess the effect of ambient features such as noise, temperature, and distractions on technical and non-technical performance. The set up can also be used to assess team dynamics during the introduction of a new procedure, such as robotic surgery. This could lead to shorter learning curves for demonstration of competence at performing the new procedure, not only for the primary surgeon, but also the rest of the surgical team. More controversially, it is also possible to assess the effect of introducing a new member to the team, such as a locum doctor or agency nurse.

Practising the skills required in a simulated environment can enable the surgical team to function in a safer and more efficient manner when the crises occur in real life, with subjective data available on the effect of CRM training in aviation and anaesthetics. ${ }^{10}{ }^{17}{ }^{37}$ However, it is also important to ensure upkeep of these skills, and regular training courses in a simulated operating theatre can allow this to happen. Studies in aviation have led to the concept of "over learning" whereby responses to crisis scenarios become automatic. ${ }^{38}$ It is not inconceivable that the simulated operating theatre could lead to the development of such expertise, without the necessity for this to be gained through real life experiences on real patients.

Hence, not only can the simulated operating theatre be used as a bridge between learning technical skills on bench top models and overall competence in the real operating room, but also as a mode of assessment of individual team members, individual teams, and the effect of external influences upon these groups. With this approach it may be possible to identify, and subsequently reduce, the high number of adverse events currently experienced in our healthcare system.

\section{ACKNOWLEDGEMENTS}

The authors would like to acknowledge Sean Mackay, Roger Kneebone, Vivek Datta, Simon Bann, Lee Edwards, Guang-Zhong Yang, Shirley Martin, and Lorraine Poore for their efforts in the development of the simulated operating theatre and allied technologies.

\section{Authors' affiliations}

R Aggarwal, S Undre, K Moorthy, C Vincent, A Darzi, Department of Surgical Oncology \& Technology, Imperial College, London, UK

Competing interests: None.

\section{REFERENCES}

1 Taffinder NJ, Smith SGT, Mair J, et al. Can a computer measure surgical precision? Reliability, validity and feasibility of the ICSAD. Surg Endosc $1999 ; 13: 81$.

2 Datta V, Mackay S, Mandalia M, et al. The use of electromagnetic motion tracking analysis to objectively measure open surgical skill in the laboratorybased model. J Am Coll Surg 2001;193:479-85.

3 Martin JA, Regehr G, Reznick R, et al. Objective structured assessment of technical skills (OSATS) for surgical residents. Br J Surg 1997;84:273-8.

4 Eubanks TR, Clements RH, Pohl D, et al. An objective scoring system for laparoscopic cholecystectomy. J Am Coll Surg 1999;189:566-74.

5 Spencer FC. Teaching and measuring surgical techniques - the technical evaluation of competence. Bull Am Coll Surg 1978;63:9-12.

6 Helmreich RL, Merritt AC, Wilhelm JA. The evolution of crew resource management training in commercial aviation. Int J Aviat Psychol 1999;9:19-32.

7 Helmreich RL, Wilhelm JA, Gregorich SE, et al. Preliminary results from evaluation of cockpit resource management training: performance ratings of flight crews. Aviat Space Environ Med 1990;61:576-9.

8 Wiener EL, Kanki BG, Helmreich RL. Cockpit resource management. San Diego, Calif: Academic Press, Inc, 1993.

9 Flin R, $\mathrm{O}^{\prime}$ Connor $\mathrm{P}$, Mearns $\mathrm{K}$. Crew resource management: improving safety in high reliability industries. Team Performance Management 2002;8:68-78.

10 Holzman RS, Cooper JB, Gaba DM, et al. Anesthesia crisis resource management: real-life simulation training in operating room crises. J Clin Anesth 1995;7:675-87.

11 Copeland GP, Jones D, Walters M. POSSUM: a scoring system for surgical audit. Br J Surg 1991; 78:355-60.

12 Vincent C, Moorthy K, Sarker SK, et al. Systems approaches to surgical quality and safety. From concept to measurement. Ann Surg 2004;239:475-82.

13 Chopra V, Bovill JG, Spierdijk J, et al. Reported significant observations during anaesthesia: a prospective analysis over an 18-month period. BrJ Anaesth 1992;68:13-17.

14 Dean B, Schachter M, Vincent CA, et al. Causes of prescribing errors in hospital inpatients: a prospective study. Lancet 2002;359:1373-8.

15 Carthey J, de Leval MR, Reason JT. The human factor in cardiac surgery: errors and near misses in a high technology medical domain. Ann Thorac Surg 2001;72:300-5.

16 Reason J. Human error: models and management. BMJ 2000;320:768-70.

17 Helmreich RL, Wilhelm JA. Outcomes of crew resource management training. Int J Aviat Psychol 1991;1:287-300.

18 Helmreich RL, Davies JM. Human factors in the operating room: interpersonal determinants of safety, efficiency and morale. In: Aitkenhead AA, ed. Bailliere's clinical anaesthesiology: safety and risk management in anaesthesia. London: Bailliere Tindall, 1996:277-96.

19 Sexton JB, Thomas EJ, Helmreich RL. Error, stress and teamwork in medicine and aviation: cross sectional surveys. BMJ 2000;320:745-9. 
20 Sandal GM. The effects of personality and interpersonal relations on crew performance during space simulation studies. The International Journal of Life Support and Biosphere Science 1999;5:226-37.

21 Sexton JB, Helmreich R. Communication and teamwork in the surgical operating room. Panel presentation at the Aerospace Medical Association Conference, Houston, Texas, 2000.

22 Krummel TM. Surgical simulation and virtual reality: the coming revolution. Ann Surg 1998;228:635-7.

23 Ponsky JL. Complications of laparoscopic cholecystectomy. Am J Surg 1991;161:393-5.

24 Bevan PG. Craft workshops in surgery. Br J Surg 1986;73:1-2.

25 Moorthy K, Munz Y, Sarker S, et al. Objective assessment of technical skills in surgery. BMJ 2003;327:1032-7.

26 Moorthy K, Munz Y, Adams S, et al. Human factors analysis of technical and team skills during procedural simulations. Br J Surg 2003;90:88-9.

27 Moorthy K, Munz Y, Forrest D, et al. Human factors analysis of crisis management skills among surgical trainees during a simulated crisis event. Br J Surg 2004;91:19.

28 Moorthy K, Munz Y, Forrest D, et al. Face validity and participants' perceptions of the value of surgical crisis management (SCM) training. Br J Surg 2004;91:84

29 Beard J, Rochester J, Thomas W. Assessment of basic surgical skills in the laboratory. Br J Surg 2003;90:90.
30 Haluck RS, Krummel TM. Computers and virtual reality for surgical education in the 21 st century. Arch Surg 2000;135:786-92.

31 Scott DJ, Bergen PC, Rege RV, et al. Laparoscopic training on bench models: better and more cost effective than operating room experience? J Am Coll Surg 2000;191:272-83.

32 Seymour NE, Gallagher AG, Roman SA, et al. Virtual reality training improves operating room performance. Ann Surg 2002;236:458-64.

33 Grantcharov TP, Kristiansen VB, Bendix J, et al. Randomised clinical trial on the transfer of laparoscopic skills from virtual environment to the operating room. Br J Surg 2004:91:146-50.

34 Kneebone RL, Nestel D, Moorthy K, et al. Learning the skills of flexible sigmoidoscopy - the wider perspective. Med Educ 2003;37:50-8.

35 Boud D, Miller N. Working with experience. Animating learning. London/ New York: Routledge, 1996

36 Helmreich RL, Schaefer HG. Team performance in the operating theatre. In: Bogner MS, ed. Human error in medicine. Hillside, NJ: Lawrence Erlbaum and Associates, 1994:225-53.

37 Helmreich RL, Foushee HC. Why cockpit resource management? Empirical and theoretical bases of human factors in training and aviation. In: Wiener $\mathrm{E}$, Kanki BG, Helmreich RL, editors, Cockpit resource management, San Diego, Calif:Academic Press, 1993:3-45.

38 Workload measurement. In: Farmer E, van Rooij J, Riemersma J, et al. Handbook of simulator-based training. Aldershot, UK: Ashgate, 1999:261-8. 